\title{
A case of Gilles de la Tourette's syndrome with midbrain involvement
}

\author{
JPWF LAKKE, JT WILMINK \\ From the Department of Neurology, University Hospital, Groningen, The Netherlands
}

SUMMARY In a 27-year-old patient with Gilles de la Tourette's syndrome a pineal tumour had been found 10 years previously and was apparently a coincidental finding. Minute calcifications were later detected around the third ventricle and the mesencephalic periaqueductal gray matter by high resolution computed tomography. It is argued that this observation may support Devinsky's speculations that midbrain involvement has pathological significance for the Gilles de la Tourette syndrome.

For many years tic conditions, including Gilles de la Tourette syndrome were attributed to psychogenic factors. The first successful treatment of a patient with Gilles de la Tourette syndrome with haldol' in the 1960s shifted attention to a neurobiochemical basis. The ability of haldol and other dopamine blocking medication to suppress tic symptoms implied abnormalities in the dopaminergic synaptic transmission system, possibly at the site of the basal ganglia. Only a few necropsy reports have appeared in the literature ${ }^{2}$ and no evidence has been found as yet for a pathological-anatomical correlate of Gilles de la Tourette syndrome. Recently Devinsky ${ }^{3}$ speculated that damage to the periaqueductal gray matter and midbrain tegmentum may be involved in Gilles de la Tourette syndrome. His reasoning was based on three observations: (1) pharmacological manipulation of the biogenic metabolism, (2) a review of symptoms and localisation of lesion in encephalitis lethargica and (3) animal studies on the anatomy of vocalisation. We present a patient with Gilles de la Tourette syndrome in whom radiological evidence was found of midbrain involvement, which may support Devinsky's hypothesis.

\footnotetext{
Address for reprint requests: Dr JPWF Lakke, University Hospital, Department of Neurology, PO Box 30.001, 9700 RB Groningen, The Netherlands.
}

Received 26 March 1985.

Accepted 10 May 1985

\section{Case report}

A 20-year-old man first attended at the age of sixteen. For the preceding three years he had been considered a hyperkinetic child, but his condition had worsened. He suffered frequent unexpected sudden contractions of his truncal musculature causing hyperextension of his body, backwards throwing of the head and a forward jerk of his pelvis. This was followed by a rapid displacement of the left leg and an outward flinging of the left arm. The short periods of instability did not cause him to fall. This complex motor tic could occur at irregulal intervals, unpredictably during activity such as walking, as well as while sitting or in the supine position. In addition explosive utterances were noted, both during the motor tic as well in the interval period. Originally an inarticulate noise, the vocal tic changed early in the course of the disease to a stereotype obscenity. Both motor and vocal tics increased during stress situations and disappeared during sleep. Previous history and family history were unremarkable. There were no indications of premature sexual maturation.

Neurological examination was normal with the exception of the tics. Routine laboratory studies, EEG, skull radiographs and echography demonstrated no abnormalities. Routine CSF examination was normal, no abnormal cells were found. HVA and 5-HIAA concentrations in CSF with probenecid were elevated; HVA 140 $\mathrm{mg} / \mathrm{ml}$ and $5 \mathrm{HIAA} 49 \mathrm{mg} / \mathrm{ml}$ (probenecid $9.0 \mu \mathrm{g} / \mathrm{ml}$ ) respectively. Treatment with haldol decreased the motor and vocal tics in the beginning of therapy but two years later the truncal jerking movements became more and more violent and a stereotactic intervention was decided upon. However, the pre-operative pneumencephalogram (fig A) showed obliteration of the pineal recess by an expanding mass with an anteroposterior diameter of some $2 \mathrm{~cm}$ (with no correction for radiological enlargement), 
which protruded into the quadrigeminal plate cistern and appeared to displace the suprapineal recess upward, while leaving the aqueduct patent. The mass contained fine granular calcification in two separate locations and was diagnosed as most probably a pinealoma.

Stereotactic therapy was not carried out and the tumour was treated with radiation $(4 \times 725 \mathrm{Rad})$. Haldol medication was increased to $20 \mathrm{mg}$ daily with useful effect. By the end of 1974 the patient started to complain of diplopia and some months later the right $\mathrm{m}$. rectus externus became paretic and the left rectus internus muscle showed some weakness. Upon downwards gaze nystagmus was seen and retraction of the right sided eyelid (Collier's sign). Pneumencephalographic examination was repeated, and showed that the size of the pineal mass had not increased. The abnormalities of ocular motility have not increased since. In the course of therapy remissions and exacerbations occurred in the severity of the tics, but in general the motor and vocal abnormalities declined. For control purposes CT was recently performed (fig B). This showed the partially calcified mass in the pineal region. Minute areas of calcification were also seen bilaterally in the adjoining posterior portion of the third ventricular wall, extending forward and laterally into the thalamus. At a lower level similar discrete calcium deposits could be seen in the brain stem at the mesencephalic level, probably located in and between the periaqueductal gray matter and the nucleus ruber.

\section{Discussion}

Devinsky proposed that the periaqueductal gray matter and midbrain tegmentum may be involved in Gilles de la Tourette syndrome, but recognised that the role of the midbrain in the pathophysiological mechanism of Gilles de la Tourette syndrome remains speculative. His eloquent reasoning is based upon (a) the correspondence of Gilles de la Tourette syndrome symptomatology with motor and vocal tics and obsessive-compulsive behaviour in encephalitis lethargica. In the latter disease the periaquaductal gray matter and midbrain tegmentum are the main sites of involvement as well as substantia migra and structures around the third ventricle. Biochemically DA, NA and 5-HT transmission are affected. (b) Midbrain stimulation in monkey, cat and birds produced a wide range of affective species-specific vocalisations, comparable with the vocal tics of Gilles de la Tourette syndrome, the latter having emotional characteristics, (c) haldol and, recently, clonidine, reducing dopaminergic and noradrenergic activity, are therapeutically effective in Gilles de la Tourette syndrome. Nuclei with these monoaminergic cells are located in the mesencephalic area.

Our findings of scattered calcification at the mesencephalic level seem to confirm Devinsky's hypothesis. However, the nature of this calcification is obscure and may be related to the pineal tumour.

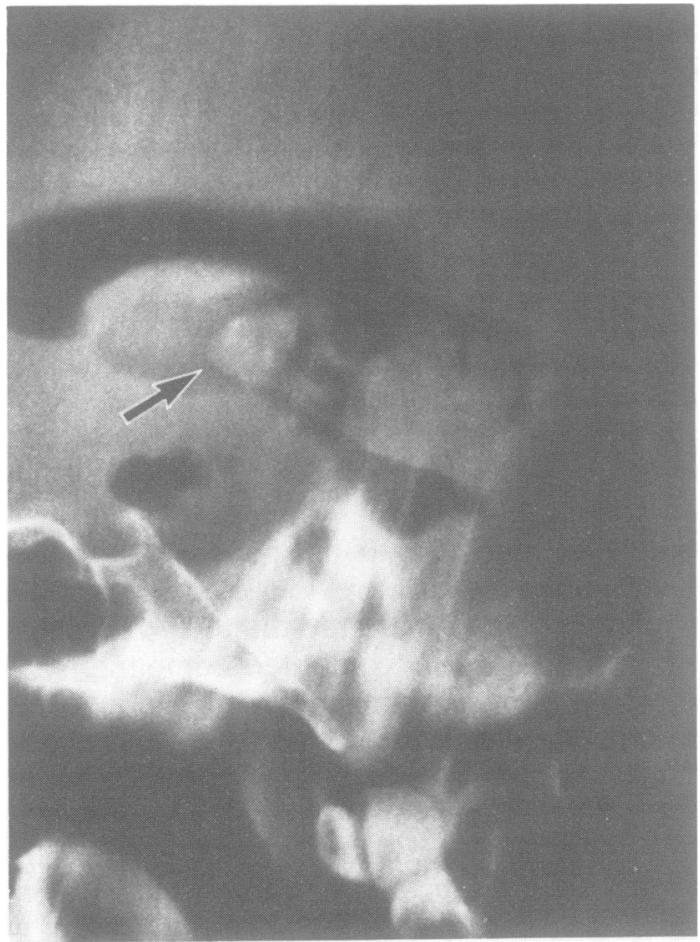

(A)

Fig 1 (A) Pneumoencephalogram, mid-sagittal tomographic section. Note air filling posterior third ventricle including suprapineal recess, also aqueduct and fourth ventricle. Pineal mass, indicated by arrow, contains fine calcification in at least two separate locations. (B) CT sections (4.5 $\mathrm{mm}$ thick) showing calcification in pineal mass $(a, b)$ in wall of third ventricle $(b)$ and in mesencephalon $(b$, $c, d)$. Sections $a, b, c$ in plane of orbito-meatal line, section $d$ more transversely oriented.

Though originally diagnosed as a pinealoma, with hindsight differential diagnostic considerations may further delineate the nature of tumour. Neoplastic metastases are unlikely in view of the elapsed time. The presence of calcification within the mass is compatible with the diagnosis of teratoma, although these tumours calcify less frequently than pinealomas. Gliomas of the posterior third ventricle rarely exhibit calcification. ${ }^{4}$ Pineal growths are histologically divided in non-parenchymal (that is germ cell tumours) and parenchymal tumours (that is true 

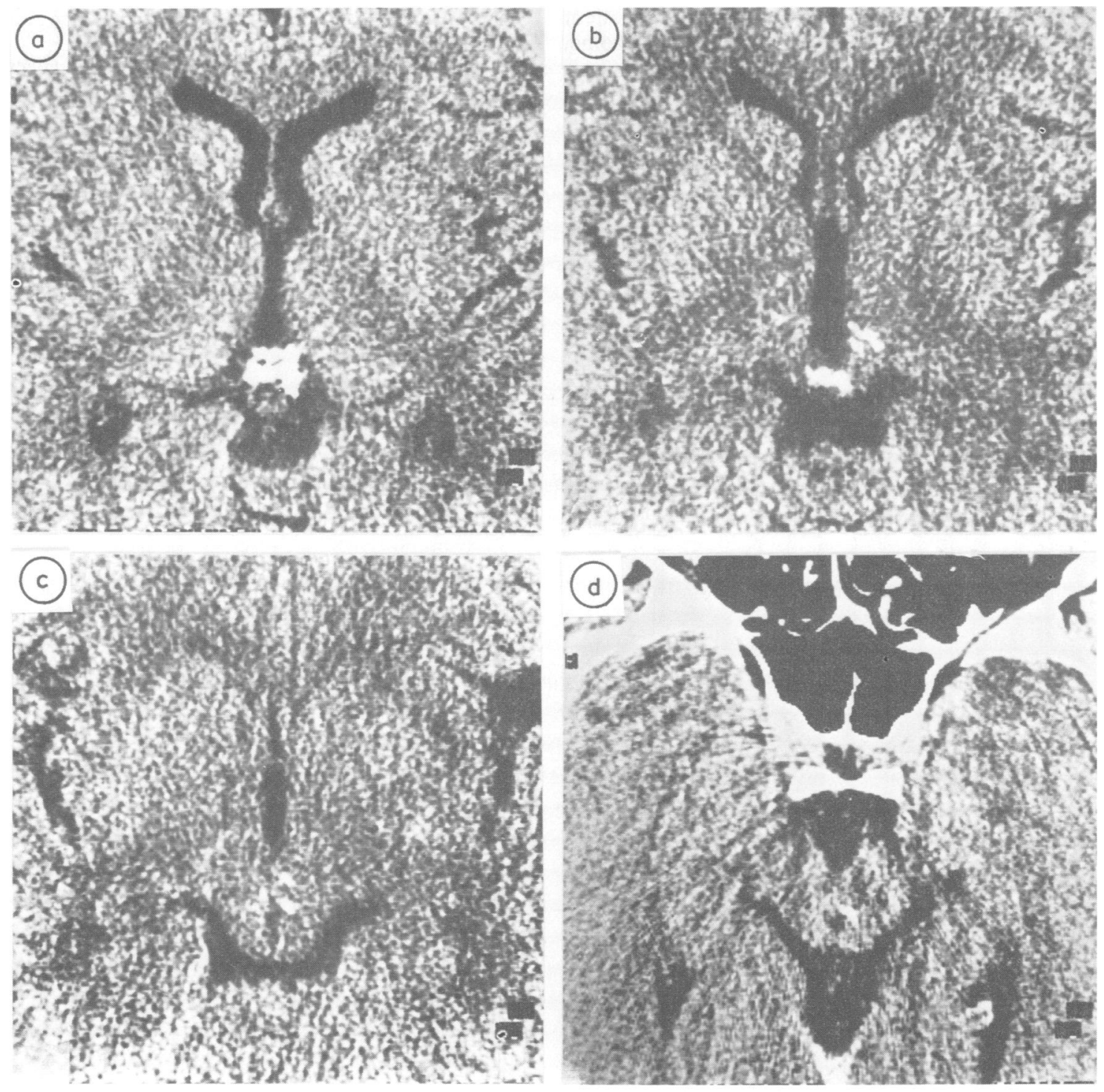

(B)

pinealomas). ${ }^{5}$ In the former group most cases of sexual precocity occur. In the latter group of pinealoma, pineoblastomas are malignant, pineocytomas much less. In contradistinction to germinomas, the pinealoma in particular has prominent calcification within the pineal mass, as has become particularly evident since computed tomography. ${ }^{6}$ These pineocytomas can be treated effectively with radiation without much chance of recurrence. We assume that our patient has a pineocytoma and radiation therapy apparently has halted tumour

growth. The calcification is not the result of radiation therapy, because it was present before commencement of therapy. However, the presence of the tumour sets our case apart from classical cases of Gilles de la Tourette syndrome, although a coincident observation is not excluded. If there is any relation between tumour, calcification and Gilles de la Tourette syndrome, then one may assume this to be a case of symptomatic Gilles de la Tourette syndrome and as such confirms the hypothesis of Devinsky. On the other hand, if a tumour in the 
pineal region is an accidental finding and our patient suffers from pure Gilles de la Tourette syndrome, the discrete calcium deposits around the posterior portion of the third ventricular wall and the brain stem at the mesencephalic level may be of aetiological significance. The likelihood of the latter assumption seems to be further confirmed by the fact that in larger series of pineal growth, ${ }^{56}$ with the exception of one case of dystonia, no abnormal movement disorders were reported. Further CT investigations in other cases of Gilles de la Tourette syndrome may clarify this intriguing problem.

\section{References}

' Seignot JN. Un cas de maladie des tics de Gilles de la
Tourette queri par le R-1625. Ann Med Psychol 1961;119:578-9.

2 Shapiro AK, Shapiro ES, Bruun RD, Sweet RD. Gilles de la Tourette Syndrome. New York, Raven Press, 1978.

${ }^{3}$ Devinsky O. Neuroanatomy of Gilles de la Tourette's Syndrome. Possible Midbrain Involvement. Arch Neurol 1983;40:508-14.

4 Taveras JM, Wood EH. Diagnostic Neuroradiology. Vol 1. Baltimore: Williams and Wilkins, 1976:443.

${ }^{5}$ Borit A. History of tumors of the pineal region. Am J Surg Pathol 1981;5:613-20.

- Wood JH, Zimmerman RA, Bruce DA, Bilaniuk LT, Norris DG, Schut L. Assessment of Management of Pineal-Region and Related Tumors. Surg Neurol 1981;16:192-210. 